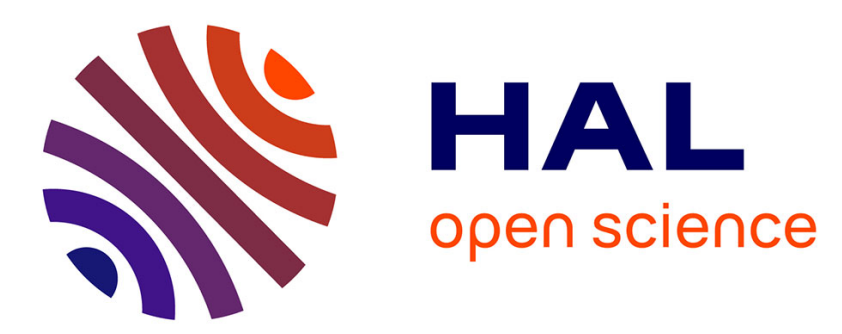

\title{
Lattice-Reduction-Aided Equalization for MIMO-FBMC Systems
}

\author{
Rostom Zakaria, Danilo Silva, Didier Le Ruyet
}

\section{To cite this version:}

Rostom Zakaria, Danilo Silva, Didier Le Ruyet. Lattice-Reduction-Aided Equalization for MIMO-FBMC Systems. IEEE Wireless Communications Letters, 2019, 8 (1), pp.101-104. 10.1109/LWC.2018.2859942 . hal-02470187

\section{HAL Id: hal-02470187 https://hal-cnam.archives-ouvertes.fr/hal-02470187}

Submitted on 7 Feb 2020

HAL is a multi-disciplinary open access archive for the deposit and dissemination of scientific research documents, whether they are published or not. The documents may come from teaching and research institutions in France or abroad, or from public or private research centers.
L'archive ouverte pluridisciplinaire HAL, est destinée au dépôt et à la diffusion de documents scientifiques de niveau recherche, publiés ou non, émanant des établissements d'enseignement et de recherche français ou étrangers, des laboratoires publics ou privés. 


\title{
Lattice-Reduction-Aided Equalization for MIMO-FBMC systems
}

\author{
Rostom Zakaria, Danilo Silva, and Didier Le Ruyet,
}

\begin{abstract}
In this paper, we propose to use a lattice reduction (LR) approach to enhance the performance of spatial multiplexing (SM) multiple-input multiple-output (MIMO) systems using filter-bank multicarrier (FBMC) modulation. It is known that lattice-reduction-aided (LRA) MIMO receivers can achieve full spatial diversity. On the other hand, the presence of the intrinsic interference in FBMC is widely regarded as a factor that prevents the system from taking advantage of spatial diversity when combined with SM-MIMO. We will show that with an adequate adaptation to FBMC modulation, the $L R$ approach can be performed in a straightforward manner. Simulation results show that an LRA equalizer allows an FBMC-MIMO system to benefit more from the available spatial diversity and offers an interesting performance gain compared to the existing solutions with reasonable complexity.
\end{abstract}

Index Terms-FBMC/OQAM, Filter-bank, MIMO, Lattice reduction, Spatial Multiplexing.

\section{INTRODUCTION}

Orthogonal Frequency Division Multiplexing (OFDM) modulation has been widely used in wireless communication systems for the past decades. The large diversity of services of the next generation wireless systems ranging from the highrate mobile broadband to ultra-high reliability communications and the multi-service dimension of next generation mobile network require better spectrum localization and robustness to time asynchronism access. Post-OFDM waveforms have to be designed to cope with these requirements. Among the different candidates, Filter-Bank Multi-Carrier (FBMC) modulation achieves the maximum spectral efficiency with a frequencylocalized pulse shape filter [1]. However, these features are obtained to the detriment of the complex orthogonality. Each demodulated data symbol is accompanied with the so-called intrinsic interference coming from the neighboring transmitted symbols. To cope with the intrinsic interference, FBMC is commonly associated with the Offset-Quadrature Amplitude Modulation (FBMC/OQAM or OFDM/OQAM) to restrict the orthogonality to the real field. The data at the receiver side is carried only by the real component of the signal. The imaginary part appears as an intrinsic interference term. The presence of this interference prevents the combination with some multiple-input/multiple-output (MIMO) techniques such

Danilo Silva is with the Department of Electrical and Electronic Engineering, Federal University of Santa Catarina, Florianópolis 88040-900, Brazil (e-mail: danilo.silva@ufsc.br).

Rostom Zakaria and Didier Le Ruyet are with Conservatoire National des Arts et Metiers, Paris, 75003, France, e-mail: (rostom.zakaria@cnam.fr, didier.le_ruyet@cnam.fr).

This work was partially funded by the $\mathrm{CNPq}$, Brazil under grants 313750/2014-9, 310343/2016-0 and 429097/2016-6, and through the french ANR project WONG5 (ANR-15-CE25-0005-02). as space-time block coding and spatial multiplexing (SM) with maximum likelihood (ML) detection [2] [3]. However, linear equalization based on zero forcing (ZF) or minimum-mean square error (MMSE) criteria can be easily performed by considering that the intrinsic interference term together with the useful data symbol form a single virtual symbol [4].

Contrary to ZF and MMSE receivers, lattice-reductionaided (LRA) equalization [5] allows to reach the optimum diversity behavior. Lattice reduction approach searches for a suitable equivalent basis for a given lattice spanned by the columns of the MIMO channel matrix. This equivalent basis has a smaller orthogonality defect, which in turn is more suitable for component-wise symbol decision. In each layer, the decided symbol is a Gaussian integer linear combination of the transmitted symbols. Different LRA algorithms have been proposed such as the Lenstra-Lenstra-Lovasz (LLL) [6] or Minkowski reduction [7] [8].

In this work, we propose to apply LRA MIMO equalization to the received FBMC/OQAM signals. To the best of our knowledge, there is no existing work that attempts to equalize the FBMC/OQAM SM-MIMO signals by using the lattice reduction approach. A major difficulty is the fact that, contrarily to the useful data symbols, the intrinsic interferences do not belong to a lattice. Therefore, it is not possible to find a Gaussian integer transformation that connects the virtual transmitted symbols (useful and interference terms) with the decided ones. In this letter, we propose to restrict the linear integer transformation to be real instead of complex. Thus, the useful data symbols and the intrinsic interferences can still be separable after the integer transformation.

The rest of the paper is organized as follows. Section II gives a brief overview of lattice-reduction-aided (LRA) MIMO equalization. The simplified system model of the MIMO FBMC/OQAM is introduced in section III. Then, we derive and present in Section IV our proposed MIMO LRA equalizer for FBMC/OQAM. Section V is dedicated for simulation results where we compare the proposed solution to the existing ones. Finally, we present concluding remarks in Section VI.

\section{LATTICE-REDUCTION-AIDED MIMO EQUALIZATION}

Assume a classical MIMO system using spatial multiplexing with $N_{t}$ transmit and $N_{r}$ receive antennas. Complex-valued symbols $s_{p}\left(p=1, \ldots, N_{t}\right)$, of variance $\sigma_{s}^{2}$ and drawn from a QAM constellation, are simultaneously radiated over the $N_{t}$ transmit antennas. Let us denote the transmitted $N_{t}$ dimensional vector as $\mathbf{s}$, the $N_{r} \times N_{t}$ channel matrix as $\mathbf{H}$, and the 
$N_{r}$ dimensional noise vector as $\mathbf{z}$. Then, the received vector $\mathbf{y}$ is expressed as:

$$
\mathbf{y}=\mathbf{H s}+\mathbf{z} .
$$

The entries of $\mathbf{z}$ are complex-valued Gaussian noise with zeromean and variance of $\sigma_{z}^{2}$. The set $\mathcal{L}(\mathbf{H})=\left\{\mathbf{H s} \mid \mathbf{s} \in \mathbb{G}^{N_{t}}\right\}^{1}$ generated by the channel matrix $\mathbf{H}$ is an $N_{t}$-dimensional lattice in $\mathbb{C}^{N_{r}}$. Lattice reduction allows to find a new basis C for the same lattice $\mathcal{L}(\mathbf{H})$ such that the new basis vectors are shorter and nearly orthogonal. To this end, the matrix $\mathbf{H}$ is factorized as

$$
\mathbf{H}=\mathbf{C A}
$$

where $\mathbf{A} \in \mathbb{G}^{N_{t} \times N_{t}}$ is a Gaussian integer linear transformation and unimodular $(|\operatorname{det}(\mathbf{A})|=1)$. Therefore, the received vector $\mathbf{y}$ is rewritten as:

$$
\mathbf{y}=\mathbf{C A} \mathbf{s}+\mathbf{z}=\mathbf{C} \overline{\mathbf{s}}+\mathbf{z}
$$

where $\overline{\mathbf{s}}=\mathbf{A s}$ is the symbol vector to be recovered at the receiver. Note that since $\mathbf{C}$ is chosen to be nearly orthogonal, recovering $\overline{\mathbf{s}}$ instead of $\mathbf{s}$ has the advantage to avoid severe noise enhancement [9]. Hence, The corresponding MMSE equalization matrix $\mathbf{B}$ is given by

$$
\mathbf{B}=\left(\mathbf{C}^{H} \mathbf{C}+\xi \mathbf{A}^{-H} \mathbf{A}^{-1}\right)^{-1} \mathbf{C}^{H}
$$

where $\xi=\sigma_{z}^{2} / \sigma_{s}^{2}$. Then, detection of $\overline{\mathbf{s}}$ is done in a component-wise manner by mapping each element of the vector By to the closest Gaussian integer. Finally, having the detection results, an estimate of the transmit symbols is obtained via $\hat{\mathbf{s}}=\mathbf{A}^{-1} \hat{\overline{\mathbf{s}}}$.

\section{MIMO FBMC/OQAM SYSTEM MODEL}

FBMC/OQAM is a multicarrier modulation where the real and imaginary parts of the QAM symbols $s_{k, 2 n}$, in each subcarrier $k$, are staggered to form a sequence of real-valued data symbols $x_{k, n}$, with $n$ being the time index. With $M$ subcarriers, the signal $x_{k, n}$ in each subcarrier $k$ is upsampled by $M / 2$ and then filtered by using a prototype filter $g[m]$ of length $K M$, where $m$ is the sampling time index and $K$ is an integer denoting the overlapping factor. The baseband transmitted signal can be then given by [2]:

$$
s[m]=\sum_{n} \sum_{k=0}^{M-1} x_{k, n} g[m-n M / 2] e^{j 2 \pi \frac{k m}{M}} e^{j \phi_{k, n}}
$$

where $\phi_{k, n}$ is a phase term. After passing through a Rayleigh selective channel, the received signal $y[m]$ is demodulated as:

$$
y_{k, n}=\sum_{m} y[m] g[m-n M / 2] e^{-j 2 \pi \frac{k m}{M}} e^{-j \phi_{k, n}}
$$

After developments, we can show that the expression of the demodulated signals $y_{k, n}$ becomes [2], [4]:

$$
y_{k, n}=h_{k, n} x_{k, n}+h_{k, n} \sum_{k^{\prime}=0}^{M-1} \sum_{n^{\prime}} x_{k^{\prime}, n^{\prime}} \Gamma_{k, n, k^{\prime}, n^{\prime}}+z_{k, n}
$$

${ }^{1} \mathbb{G}$ stands for the set of Gaussian integers and is defined as $\mathbb{G}=\mathbb{Z}+j \mathbb{Z}$. where $z_{k, n}$ and $h_{k, n}$ are, respectively, the noise term at the demodulator output and the channel frequency response. The function $\Gamma_{k, n, k^{\prime}, n^{\prime}}$ is the so-called transmultiplexer impulse response and is given by [2]:

$$
\begin{array}{rl}
\Gamma_{k, n, k^{\prime}, n^{\prime}}=\sum_{m} & g[m-n M / 2] g\left[m-n^{\prime} M / 2\right] e^{j \frac{2 \pi m}{M}\left(k-k^{\prime}\right)} \\
& \times e^{j \phi_{k, n}-j \phi_{k^{\prime}, n^{\prime}}}
\end{array}
$$

The phase term $\phi_{k, n}$ is chosen so that $\mathfrak{R e}\left\{\Gamma_{k, n, k^{\prime}, n^{\prime}}\right\}=0$ $\forall(k, n) \neq\left(k^{\prime}, n^{\prime}\right)$. Therefore, the intrinsic interference term in (7) is purely imaginary and the equation can be rewritten as [2]:

$$
y_{k, n}=h_{k, n}\left(x_{k, n}+j u_{k, n}\right)+z_{k, n}
$$

where the term $u_{k, n}$ is real-valued.

In the MIMO spatial multiplexing case with $N_{t}$ transmit and $N_{r}$ receive antennas, real-valued data symbol $x_{k, n}^{(p)}$ are transmitted at the $p$ th antenna, with $p=1, \ldots, N_{t}$. The demodulated symbol $y_{k, n}^{(q)}$ at the $q$ th antenna and frequencytime position $(k, n)$ is expressed as:

$$
y_{k, n}^{(q)}=\sum_{p=1}^{N_{t}} h_{k, n}^{(q p)}\left(x_{k, n}^{(p)}+j u_{k, n}^{(p)}\right)+z_{k, n}^{(q)}
$$

In a matrix form and skipping the frequency-time indices $(k, n)$, the FBMC/OQAM MIMO system model is given by:

$$
\mathbf{y}=\mathbf{H}(\mathbf{x}+j \mathbf{u})+\mathbf{z}
$$

where $\mathbf{x} \in \mathbb{R}^{N_{t} \times 1}$ is the vector of transmitted symbols, $\mathbf{u} \in$ $\mathbb{R}^{N_{t} \times 1}$ is the corresponding FBMC intrinsic interference, $\mathbf{z} \in$ $\mathbb{C}^{N_{r} \times 1}$ is zero-mean circularly-symmetric complex Gaussian noise, $\mathbf{H} \in \mathbb{C}^{N_{r} \times N_{t}}$ is the channel matrix, and $\mathbf{y} \in \mathbb{C}^{N_{r} \times 1}$ is the vector of received symbols.

\section{LRA EQUALIZATION FOR FBMC/OQAM}

In this section, we propose an LRA equalization adapted for FBMC/OQAM MIMO systems. In the context of spatial multiplexing, the transmit data symbols in the vector $\mathbf{x}$ are assumed to be uncorrelated and have the same variance $\sigma_{x}^{2}$. Consequently and according to (7), the entries of the interference vector $\mathbf{u}$ are also uncorrelated and have the same variance $\sigma_{u}^{2}$. It is shown in [10] that in FBMC/OQAM the intrinsic interference and the data symbol have the same variance (i.e $\sigma_{x}^{2}=\sigma_{u}^{2}$ ). Moreover, the data symbols in each transmit antenna and for different subcarriers and time indices are assumed to be uncorrelated. Hence, the vectors $\mathbf{x}$ and $\mathbf{u}$ are pairwise orthogonal. That is, we have $E\left[\mathbf{x x}^{T}\right]=E\left[\mathbf{u u}^{T}\right]=\sigma_{x}^{2} \mathbf{I}$ and $E\left[\mathbf{x u}^{T}\right]=\mathbf{0}$. As for the noise term $\mathbf{z}$, it is assumed to be i.i.d. with variance $\sigma^{2}$ per real/imaginary component, i.e., $E\left[\mathbf{z z}^{H}\right]=2 \sigma^{2} \mathbf{I}$, and that it is orthogonal to both $\mathbf{x}$ and $\mathbf{u}$, i.e., $E\left[\mathbf{z x}^{T}\right]=E\left[\mathbf{z u}^{T}\right]=\mathbf{0}$. We define $\gamma=N_{t} \sigma_{x}^{2} / \sigma^{2}$ as the average signal to noise ratio $(\mathrm{SNR})$ at each receive antenna.

As mentioned in Section II, LRA equalization is based on factorizing the matrix channel $\mathbf{H}$ as in (2), which yields

$$
\mathbf{y}=\mathbf{C A}(\mathbf{x}+j \mathbf{u})+\mathbf{z}
$$


Then, after equalizing the reduced channel $\mathbf{C}$, decisions should be made to recover the vector $\mathbf{A}(\mathbf{x}+j \mathbf{u})$. However, due to the nature of the interference vector $\mathbf{u}$, decisions cannot be made on $\mathbf{A}(\mathbf{x}+j \mathbf{u})$ as it does not belong to a regular lattice. To overcome this issue, we propose to restrict $\mathbf{A}$ to be (real) integer matrix instead of Gaussian integer one. This allows us to recover $\mathbf{A x}$ by making decisions on $\mathfrak{R e}\{\mathbf{A}(\mathbf{x}+j \mathbf{u})\}$.

For a given $\mathbf{A} \in \mathbb{Z}^{N_{t} \times N_{t}}$, we apply an equalization matrix $\mathbf{B}$ to the received vector $\mathbf{y}$, giving

$$
\begin{aligned}
\mathbf{B y} & =\mathbf{B H}(\mathbf{x}+j \mathbf{u})+\mathbf{B z} \\
& =\mathbf{A}(\mathbf{x}+j \mathbf{u})+(\mathbf{B H}-\mathbf{A})(\mathbf{x}+j \mathbf{u})+\mathbf{B} \mathbf{z} .
\end{aligned}
$$

Taking the real part, we have

$$
\begin{aligned}
\mathbf{r} & =\mathfrak{R e}\{\mathbf{B} \mathbf{y}\} \\
& =\mathbf{A} \mathbf{x}+\mathbf{z}_{\mathrm{eff}}
\end{aligned}
$$

where

$$
\mathbf{z}_{\text {eff }}=\mathfrak{R e}\{(\mathbf{B H}-\mathbf{A})(\mathbf{x}+j \mathbf{u})+\mathbf{B z}\}
$$

is the so-called effective noise. The objective is to minimize the effective noise variance in each layer. Therefore, the remaining task is to select matrices $\mathbf{B}$ and $\mathbf{A}$ that minimize the diagonal entries of the effective noise covariance matrix $\mathbf{K}_{\mathrm{eff}}=E\left[\mathbf{z}_{\mathrm{eff}} \mathbf{z}_{\mathrm{eff}}^{T}\right]$.

To begin with, let us write $\mathbf{z}=\mathbf{z}_{\mathrm{re}}+j \mathbf{z}_{\mathrm{im}}, \mathbf{H}=\mathbf{H}_{\mathrm{re}}+j \mathbf{H}_{\mathrm{im}}$ and $\mathbf{B}=\mathbf{B}_{\text {re }}-j \mathbf{B}_{\mathrm{im}}$, where all the components are real. We can derive $\mathbf{z}_{\mathrm{eff}}$ as follows:

$$
\begin{aligned}
\mathbf{z}_{\mathrm{eff}}= & \mathfrak{R e}\{(\mathbf{B H}-\mathbf{A})(\mathbf{x}+j \mathbf{u})+\mathbf{B} \mathbf{z}\} \\
= & (\mathfrak{R e}\{\mathbf{B H}\}-\mathbf{A}) \mathbf{x}-\mathfrak{I m}\{\mathbf{B} \mathbf{H}\} \mathbf{u}+\mathfrak{R e}\{\mathbf{B} \mathbf{z}\} \\
= & \left(\mathbf{B}_{\mathrm{re}} \mathbf{H}_{\mathrm{re}}+\mathbf{B}_{\mathrm{im}} \mathbf{H}_{\mathrm{im}}-\mathbf{A}\right) \mathbf{x}-\left(\mathbf{B}_{\mathrm{re}} \mathbf{H}_{\mathrm{im}}-\mathbf{B}_{\mathrm{im}} \mathbf{H}_{\mathrm{re}}\right) \mathbf{u} \\
& +\mathbf{B}_{\mathrm{re}} \mathbf{z}_{\mathrm{re}}+\mathbf{B}_{\mathrm{im}} \mathbf{z}_{\mathrm{im}} \\
= & (\overline{\mathbf{B}} \overline{\mathbf{H}}-\overline{\mathbf{A}}) \overline{\mathbf{x}}+\overline{\mathbf{B}} \overline{\mathbf{z}}
\end{aligned}
$$

where

$$
\begin{array}{lll}
\overline{\mathbf{B}}=\left[\begin{array}{ll}
\mathbf{B}_{\mathrm{re}} & \mathbf{B}_{\mathrm{im}}
\end{array}\right], & \overline{\mathbf{H}}=\left[\begin{array}{cc}
\mathbf{H}_{\mathrm{re}} & -\mathbf{H}_{\mathrm{im}} \\
\mathbf{H}_{\mathrm{im}} & \mathbf{H}_{\mathrm{re}}
\end{array}\right], & \overline{\mathbf{z}}=\left[\begin{array}{c}
\mathbf{z}_{\mathrm{re}} \\
\mathbf{z}_{\mathrm{im}}
\end{array}\right] \\
\overline{\mathbf{x}}=\left[\begin{array}{l}
\mathbf{x} \\
\mathbf{u}
\end{array}\right], & \overline{\mathbf{A}}=\left[\begin{array}{ll}
\mathbf{A} & \mathbf{0}
\end{array}\right] .
\end{array}
$$

Now, since $E\left[\overline{\mathbf{x}} \overline{\mathbf{x}}^{T}\right]=\sigma_{x}^{2} \mathbf{I}, E\left[\overline{\mathbf{z}} \overline{\mathbf{z}}^{T}\right]=\sigma^{2} \mathbf{I}$, and $E\left[\overline{\mathbf{x}} \overline{\mathbf{z}}^{T}\right]=$ $\mathbf{0}$, we can write the covariance matrix of the effective noise as follows:

$$
\begin{aligned}
\mathbf{K}_{\mathrm{eff}} & =E\left[\mathbf{z}_{\mathrm{eff}} \mathbf{z}_{\mathrm{eff}}^{T}\right] \\
& =E\left[((\overline{\mathbf{B}} \overline{\mathbf{H}}-\overline{\mathbf{A}}) \overline{\mathbf{x}}+\overline{\mathbf{B}} \overline{\mathbf{z}})((\overline{\mathbf{B}} \overline{\mathbf{H}}-\overline{\mathbf{A}}) \overline{\mathbf{x}}+\overline{\mathbf{B}} \overline{\mathbf{z}})^{T}\right] \\
& =(\overline{\mathbf{B}} \overline{\mathbf{H}}-\overline{\mathbf{A}})(\overline{\mathbf{B}} \overline{\mathbf{H}}-\overline{\mathbf{A}})^{T} \sigma_{x}^{2}+\overline{\mathbf{B}} \overline{\mathbf{B}}^{T} \sigma^{2}
\end{aligned}
$$

That is,

$$
\mathbf{K}_{\mathrm{eff}} / \sigma^{2}=(\overline{\mathbf{B}} \overline{\mathbf{H}}-\overline{\mathbf{A}})(\overline{\mathbf{B}} \overline{\mathbf{H}}-\overline{\mathbf{A}})^{T} \frac{\gamma}{N_{t}}+\overline{\mathbf{B}} \overline{\mathbf{B}}^{T} .
$$

As it is known from [11], for a fixed integer matrix $\mathbf{A}$ the diagonal entries of $\mathbf{K}_{\text {eff }}$ are minimized by choosing

$$
\overline{\mathbf{B}}=\overline{\mathbf{A}} \overline{\mathbf{H}}^{T}\left(N_{t} \gamma^{-1} \mathbf{I}+\overline{\mathbf{H}} \overline{\mathbf{H}}^{T}\right)^{-1}
$$

or equivalently

$$
\mathbf{B}=\mathbf{A} \mathbf{H}^{H}\left(N_{t} \gamma^{-1} \mathbf{I}+\mathbf{H} \mathbf{H}^{H}\right)^{-1} .
$$

By plugging (18) into (17), we can show that [11]

$$
\begin{aligned}
\mathbf{K}_{\text {eff }} / \sigma^{2} & =\overline{\mathbf{A}}\left(N_{t} \gamma^{-1} \mathbf{I}+\overline{\mathbf{H}}^{T} \overline{\mathbf{H}}\right)^{-1} \overline{\mathbf{A}}^{T} \\
& =\overline{\mathbf{A}} \overline{\mathbf{M}} \overline{\mathbf{A}}^{T},
\end{aligned}
$$

where

$$
\overline{\mathbf{M}}=\left(N_{t} \gamma^{-1} \mathbf{I}+\overline{\mathbf{H}}^{T} \overline{\mathbf{H}}\right)^{-1}
$$

We can also rewrite expression (19) as:

$$
\mathbf{K}_{\mathrm{eff}} / \sigma^{2}=\mathbf{A M A}^{T}
$$

with

$$
\mathbf{M}=\mathfrak{R e}\left\{\left(N_{t} \gamma^{-1} \mathbf{I}+\mathbf{H}^{H} \mathbf{H}\right)^{-1}\right\} .
$$

Therefore, the effective noise variance in each layer $i$ (which is the $i$ th diagonal element of $\mathbf{K}_{\text {eff }}$ ) is given by

$$
\sigma_{\mathbf{z}_{\text {eff }}(i)}^{2}=\sigma^{2} \mathbf{a}_{i}^{T} \mathbf{M} \mathbf{a}_{i}
$$

where $\mathbf{a}_{i}$ is the $i$ th column vector of $\mathbf{A}^{T}$. Since $\mathbf{M}$ is a real-valued and symmetric positive definite matrix, we can write $\mathbf{M}=\mathbf{L}^{T} \mathbf{L}$ for some $\mathbf{L} \in \mathbb{R}^{N_{t} \times N_{t}}$ via Cholesky decomposition. Hence, (21) becomes

$$
\sigma_{\mathbf{z}_{\text {eff }}(i)}^{2}=\sigma^{2}\left\|\mathbf{L} \mathbf{a}_{i}\right\|^{2} .
$$

Therefore, to construct the optimal integer matrix $\mathbf{A}$, we have to obtain $N_{t}$ vectors $\mathbf{a}_{i}$ which result in the first $N_{t}$ smaller norms $\left\|\mathbf{L} \mathbf{a}_{i}\right\|$ along with the unimodular property on $\mathbf{A}$ (i.e. $\operatorname{det}(\mathbf{A})= \pm 1$ ) [8]. This problem corresponds to finding a shortest basis for the lattice generated by $\mathbf{L}$ [11], which can be solved by well-known Minkowski-reduction algorithms [12], as well as Lagrange-Gauss reduction (LGR) in the special case of $N_{t}=2$ [13]. Another widely known algorithm is the socalled LLL (Lenstra-Lenstra-Lovasz) algorithm [6] which can find a reasonably close to optimal solution in polynomial time.

\section{Simulation Results}

We now evaluate the performance of the proposed receiver architecture in the uncoded case where the transmitted symbols belong to the finite constellation 2-PAM (corresponding to a 4-OQAM complex modulation in FBMC).

In figure 1, we have compared the bit error rate performance of the LGR-based algorithm over an $N_{t}=N_{r}=2$ MIMO Rayleigh fading channel. We have assumed perfect channel state information and that the channels follow the ITU Pedestrian-A [14] channel model. For comparison, we have also plotted the performance of the ZF and MMSE detectors for OFDM. It is known from [4] that each of $\mathrm{ZF}$ and MMSE achieves the same performance for both OFDM and FBMC schemes. We have also shown the performance of ML and LGR-based algorithm for OFDM modulation, and the performance of the so-called MMSE-ML receiver which is proposed for MIMO-FBMC in [3].

As we can see in the figure, for BER $=10^{-2}$, the LGR-based receiver in FBMC achieves a gain of about $2 \mathrm{~dB}$ with respect to the MMSE detector. However, compared to the LGRbased algorithm for OFDM modulation, when considering FBMC modulation, the proposed LGR-based receiver does not achieve the maximum spatial diversity of 2 . This is mainly 




Fig. 1. The BER performance of ZF, MMSE, ML and LGR based algorithms for $2 \times 2$ MIMO-OFDM and MIMO-FBMC modulations

due to the restriction of the search domain of the matrix A. Interestingly, LGR-based receiver exhibits the same BER performance as the MMSE-ML. In addition, it is worth noting that MMSE-ML has a higher complexity since it performs an exhaustive search in the ML stage [3].

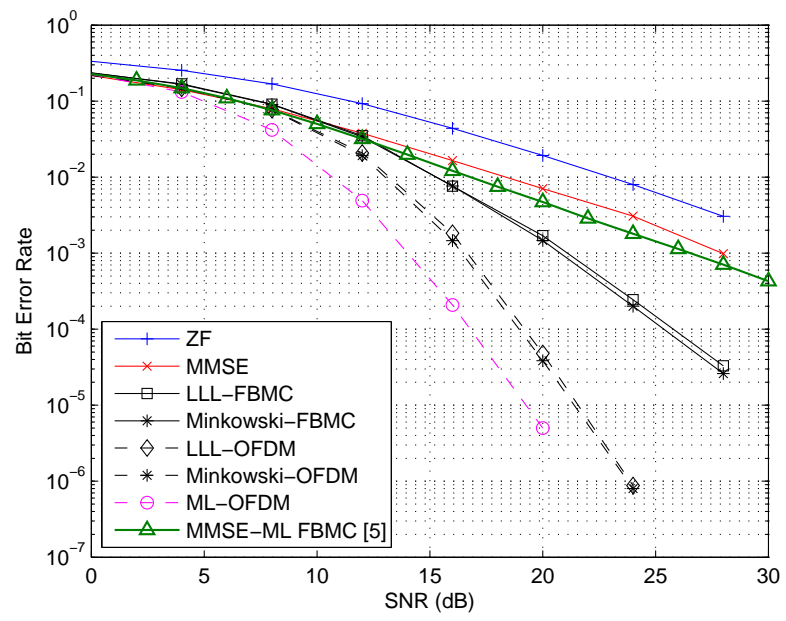

Fig. 2. The BER performance of ZF, MMSE, ML, LLL and Minkowski based algorithms for $4 \times 4$ MIMO-OFDM and MIMO-FBMC modulations

In figure 2, we have considered the case of a MIMOFBMC transmission over a Rayleigh fading channel with $N_{t}=N_{r}=4$ and 4-OQAM complex modulation. We have compared the performance of the LLL based and Minkowski based receivers with respect to the one of the MMSE detector. The LLL based and Minkowski based receivers have almost the same performance and outperform the MMSE and MMSEML receivers with a gain at $\mathrm{BER}=10^{-3}$ of about $7 \mathrm{~dB}$ and $5.5 \mathrm{~dB}$, respectively. It can be also noted that LLL and Minkowski based receivers achieve a diversity of about 2, while MMSE-ML has only a diversity of one even in a $4 \times 4$ MIMO system. For comparison, we have also added the performance of LLL and Minkowski based receivers using
OFDM modulation which achieve the full available diversity of 4. Therefore, we observe again that LR based receivers in FBMC can achieve half of the available diversity in SMMIMO configuration. This seems to be due to the restriction to real numbers on the entries of $\mathbf{A}$.

\section{CONCLUSIONS}

In this work, we have considered the lattice-reductionaided (LRA) MIMO equalization for FBMC/OQAM. We have shown that the FBMC/OQAM intrinsic interference prevents the receiver from achieving full diversity that LRA is supposed to. Indeed, since the interference does not belong to a lattice, the choice of the transformation matrix should be restricted to the set of "real" integer matrices in order to maintain the interference and the data symbols separate. Clearly, this restriction sacrifices part of the degrees of freedom and limits the achieved spatial diversity. Simulation results show that the proposed receiver offers a significant performance improvement compared to the MMSE receiver. This performance gain becomes more pronounced as the size of the MIMO system increases.

\section{REFERENCES}

[1] M. Bellanger et al., "FBMC physical layer: a primer", website: www.ictphydyas.org, June 2010.

[2] A. I. Pérez-Neira et al., "MIMO Signal Processing in Offset- QAM Based Filter Bank Multicarrier Systems", IEEE Transactions on Signal Processing, 64(21):5733-5762, nov 2016.

[3] R. Zakaria, D. Le Ruyet, and M. Bellanger, "Maximum likelihood detection in spatial multiplexing with FBMC," in European Wireless Conference, Lucca, Italy, April 2010.

[4] M. El Tabach, J.-P. Javaudin, and M. Hélard, "Spatial data multiplexing over OFDM/OQAM modulations," in Proc.International Conference on Communications (ICC), Glasgow, Scotland, June 2007.

[5] H. Yao and G. Wornell, "Lattice-reduction-aided detectors for MIMO communication systems" in Proc. IEEE Global Telecommunications Conference (GLOBECOM), p. 424-428, 2002.

[6] A. K. Lenstra, H. W. Lenstra, and L. Lovasz, "Factoring Polynomials with Rational Coefficients", in Math. Ann, vol. 261, pp. 515-534, 1982.

[7] S.Stern and R. Fischer. "V-BLAST in lattice reduction and integer forcing.", Proc. IEEE International Symposium on Information Theory (ISIT), Aachen, Germany, June 2017.

[8] A.Sakzad, J. Harshan and E. Viterbo, "Integer-forcing MIMO linear receivers based on lattice reduction", IEEE transactions on wireless communications, 12(10), 4905-4915, 2013.

[9] R. Fischer, M. Cyran, S. Stern, "Factorization approaches in latticereduction-aided and integer-forcing equalization", in Proc. International Zurich Seminar on Communications, Zurich, 2016.

[10] C. Lélé, P. Siohan, R. Legouable, J.-P. Javaudin, "Preamble-based channel estimation techniques for OFDM/OQAM over the powerline", IEEE International Symposium on Power Line Communications and Its Applications (ISPLC), pp. 59-64, Mar. 2007.

[11] J Zhan, B. Nazer, U. Erez and M. Gaspar, "Integer-forcing linear receivers", IEEE Transactions on Information Theory, vol. 60, no 12 , p. 7661-7685, 2014

[12] W. Zhang, S. Qiao and Y. Wei, "HKZ and Minkowski Reduction Algorithms for Lattice-Reduction-Aided MIMO Detection," in IEEE Transactions on Signal Processing, vol. 60, no. 11, pp. 5963-5976, Nov. 2012.

[13] M.R. Bremner, Lattice basis reduction: an introduction to the LLL algorithm and its applications, CRC Press, Boca Raton, FL, 2012.

[14] ITU-R M.1225. "Guidelines for evaluations of radio transmission technologies for IMT-2000," 1997 